gr-qc/0108071

\title{
Dimensional Dependence of Black Hole Formation in Self-Similar Collapse of Scalar Field
}

\author{
Hyeong-Chan $\operatorname{Kim}^{a *}$, Sei-Hoon Moon $^{b} \dagger$ and Jae Hyung Yee ${ }^{a, b} \ddagger$ \\ ${ }^{a}$ Institute of Basic Science, Yonsei University, Seoul 120-749, Korea \\ ${ }^{b}$ Institute of Physics and Applied Physics, Yonsei University, Seoul 120-749, Korea
}

(November 5, 2018)

\begin{abstract}
We study classical and quantum self-similar collapses of a massless scalar field in higher dimensions, and examine how the increase in the number of dimensions affects gravitational collapse and black hole formation. Higher dimensions seem to favor formation of black hole rather than other final states, in that the initial data space for black hole formation enlarges as dimension increases. On the other hand, the quantum gravity effect on the collapse lessens as dimension increases. We also discuss the gravitational collapse in a brane world with large but compact extra dimensions.
\end{abstract}

Keywords: gravitational collapse, self-similarity, black hole, quantum gravity, higher dimension, large extra dimensions.

PACS numbers: 04.50.+h; 04.70.-s; 11.10.Kk; 11.25.Mj; 11.27.+d

*E-mail: hckim@phya.yonsei.ac.kr

†E-mail: jeollo@phya.yonsei.ac.kr

$\ddagger$ E-mail: jhyee@phya.yonsei.ac.kr 


\section{INTRODUCTION}

Since the Choptuik's discovery [1] of critical phenomena in gravitational collapse of a scalar field, there have been much attention to the gravitational collapse just at the threshold of black hole formation. Similar phenomena to Choptuik's results were quickly found in other systems numerically [2]- |11] and analytically [12]- |19] too.1 suggesting that they were limited neither to scalar field matter nor to spherical symmetry. There are two possible final outcomes of gravitational collapses, distinguished by whether or not a black hole is formed in the course of evolution. Which end state is realized depends on initial data specified by a control parameter $p$ that characterizes the gravitational interaction strength in the ensuing evolution. For $p<p^{*}$ the gravitational field is too weak to form a black hole, while for $p>p^{*}$ a black hole is produced. The critical solution with $p=p^{*}$ just at the threshold of black hole formation acts as an intermediate attractor for nearby initial conditions and has an additional symmetry: discrete or continuous self-similarity.

While critical gravitational collapse has been originally studied in four spacetime dimensions, there have been several attempts to study it in higher-dimensional spacetime [21][27]. As most attempts (including string theory) to unify gravity with other forces require additional spatial dimensions, it may be meaningful to consider higher dimensional collapse problem. Although the extra dimensions are compactified to a small size and usually unobservable at low energy, the extra dimensions may be opened in the large energy limit of the Planck scale, which is relevant as we approach singularity in gravitational collapse.

Moreover, in recent years there have been explosive interests in the large extra dimension scenario [32, in which the size of extra dimensions can be as big as sub-millimeter and the fundamental scale of the higher-dimensional gravity as low as a $\mathrm{TeV}$ range. An exciting consequence of this scenario is the possibility of production of black hole at realistic future colliders 34. The Schwarzschild radius of so produced black hole will be much less than the size of extra dimensions because the mass of the black hole is typically of the order of the higher-dimensional Planck scale of TeV. Therefore, in this scheme the gravitational collapse will be essentially higher-dimensional and quantum gravitational. We can also expect the formation of black holes with much larger mass than the fundamental mass scale, but with smaller Schwarzschild radius than the size of extra dimensions. Then, black holes can be well treated as general relativistic objects in higher dimensions. Such a black hole may be formed from a macroscopic initial distribution of matter prepared in four-dimensional world, but whether black hole are formed from the given initial data depends on the number of extra dimensions, because the final stage of collapse will be entirely higher-dimensional. Therefore, we expect that one can determine the number of large extra dimensions by investigating the gravitational collapse and the dimensional dependence of black hole formation, at least, in principle. In this sense it will be valuable to study the gravitational collapse in higher dimensions and the dimensional dependence of black hole formation.

In the next section, we shall start with a brief summary of Frolov's works [22], which treat the higher-dimensional scalar field collapse with restriction to the spherical symmetry and self-similarity and examine an analytic solution. We then examine the role of dimensionality of spacetime in the gravitational collapse and black hole formation. Higher dimensions seem

\footnotetext{
${ }^{1}$ See the excellent review by Gundlach [20], and more references there in.
} 
to favor black hole in that, as dimension increases, the critical value of the control parameter decreases and so the initial data space for black hole formation enlarges, while the size of the apparent horizon increases near the critical collapse.

We will also consider, in Section III, the quantum gravity effects on the collapse of the scalar field by extending the work of Ref. [29,30] to higher dimensions. Since the mass of the black hole near the critical collapse can be arbitrarily small, the quantum gravity effects may play a significant role in such collapse. We also examine the role of spacetime dimensionality in such quantum collapse. The quantum gravity effects on the collapse decrease as the spacetime dimension increases. Finally, in the last section, we discuss the gravitational collapse in the brane world with large extra dimensions.

\section{DIMENSIONAL DEPENDENCE OF GRAVITATIONAL COLLAPSE}

We begin this section with a short review of Frolov's solutions of Ref. [22] for gravitational collapse of a minimally coupled scalar field in $n$ dimensions. The action of the system is

$$
S=\int_{M} d^{n} x \sqrt{-g}\left[\frac{1}{2 \kappa} R-\frac{1}{2}(\nabla \phi)^{2}\right]+S_{\text {surface }}
$$

where $\kappa \equiv 8 \pi M_{*}^{-(n-2)}$ and $M_{*}$ is the fundamental scale of the $n$-dimensional gravity theory. The scalar field $\phi$ has a mass dimension of $(n-2) / 2$. With the spherical symmetry and the self-similarity ansätz

$$
d s^{2}=e^{2 \sigma(\tau)}\left(-x^{2} d \tau^{2}+d x^{2}\right)+r^{2} d \Omega_{n-2}^{2}, \quad r=x y(\tau) \text { and } \phi=\phi(\tau),
$$

after taking into account the surface term the Einstein-Hilbert action is reduced to

$$
S_{\text {red }}=V_{n-2} \ell_{*}^{n-2} \int d \chi d \tau y^{n-4}\left[\frac{y^{2} \ddot{\sigma}}{\kappa(n-2)}+\frac{n-3}{2 \kappa}\left(-\dot{y}^{2}-y^{2}+e^{2 \sigma}\right)+\frac{y^{2} \dot{\phi}^{2}}{2(n-2)}\right],
$$

where the dots denote derivative with respect to $\tau$ and we have introduced a dimensionless parameter $\chi \equiv x^{n-2} / \ell_{*}^{n-2}$, where $\ell_{*}\left(\equiv M_{*}^{-1}\right)$ is the fundamental length. We have integrated out the angular variables, and $V_{n-2}$ is the volume of $(n-2)$-dimensional unit sphere, i.e., $V_{n-2} \equiv \int d \Omega_{n-2}=2 \pi^{(n-1) / 2} / \Gamma[(n-1) / 2]$. From this reduced action, one can easily see that $\sigma=$ constant, and after appropriate rescaling of coordinates $\sigma$ can be set to zero. The classical equations of motion for $\phi$ and $y$ are then given by

$$
\begin{aligned}
\dot{\phi} & =\sqrt{\frac{(n-2)(n-3) c}{\kappa}} \frac{1}{y^{(n-2)}}, \\
\dot{y}^{2} & =y^{2}-1+\frac{c}{y^{2(n-3)}} .
\end{aligned}
$$

Here, $c(>0)$ is a dimensionless integration constant and characterizes the initial distribution of the massless scalar field. It physically represents the gravitational interaction strength in the collapse and its inverse gives the measure of the inhomogeneity of the collapse.[

\footnotetext{
${ }^{2}$ The constant $c$ does the same role with parameters $\lambda$ and $\zeta_{0}$ of Refs. [26] and [28], respectively, in which self-similarly collapsing null fluid and dust are examined in different spacetime dimensions.
} 
From Eq. (5), we see that the collapsing system can be replaced by the system of a particle (living in $y$ space) with zero energy under the influence of the potential

$$
V_{\mathrm{cl}}(y)=\frac{1}{2}\left(1-y^{2}\right)-\frac{c}{2} y^{-2(n-3)} .
$$

The collapse is then parallel to the motion of a particle which starts from infinity $(y=\infty)$ and rolls toward zero $(y=0)$.

To see when a black hole is formed, we introduce the local mass function $M(\tau, x)$ such as

$$
1-\frac{2 \kappa}{(n-2) V_{n-2}} \frac{M(\tau, x)}{r^{n-3}}=(\nabla r)^{2} .
$$

Clearly, if $n=4$, this reduces to the one usually used in four-dimensional spacetime, and if the spacetime is static, it gives the correct mass of Schwarzschild black holes in $n$-dimensions [31]. We can then localize the apparent horizons given by $(\nabla r)^{2}=0$, which translates to $\dot{y}^{2}-y^{2}=0$. With this fact, from Eq.(5) we see that the black hole is formed if the value of $y$ reaches

$$
y_{\mathrm{AH}}=c^{1 / 2(n-3)} .
$$

There are three types of solutions depending on the value of the constant $c$, i.e., subcritical $\left(c<c^{*}\right)$, critical $\left(c=c^{*}\right)$ and supercritical $\left(c>c^{*}\right)$ solutions, where $c^{*}$ is a critical value of $c$ characterized by potential having second order zero, i.e., $V_{\mathrm{cl}}=V_{\mathrm{cl}}^{\prime}=0$ at $y^{*}=\sqrt{(n-3) /(n-2)}$, and it is given by

$$
c_{n}^{*}=\frac{1}{(n-3)}\left[\frac{n-3}{n-2}\right]^{n-2} .
$$

In the supercritical case where $V_{\mathrm{cl}}<0$ everywhere, the value of $y$ reaches $y_{\mathrm{AH}}$ and a black hole is formed by the collapse. On the other hand, in the subcritical case where there exists a region with $V_{\mathrm{cl}}>0$, the value of $y$ turns around at the turning point and never reaches $y_{\mathrm{AH}}$, which is located in the forbidden region. In other words, the scalar field collapses, interacts and disperses leaving behind a flat spacetime without forming a black hole. In the critical case $y$ takes infinite time to reach $y_{\mathrm{AH}}$ and $y=0$, and the spacetime is asymptotically flat but contains a null, scalar-curvature singularity.

The local mass function of the black hole formed in the supercritical collapse is given from Eq.(17) by

There, $\lambda$ and $\zeta_{0}$ characterize the initial distributions of null fluid and dust in each collapses. Their inverses also give the measure of the inhomogeneity of the collapse. For large values of their inverses one has highly inhomogeneous collapses where the outer shells collapse much later than the central ones [36]. The common characters of the parameters $c, \lambda$ and $\zeta_{0}$ can be easily observed from comparing the energy densities of the systems. In fact, we could choose other parameters that prescribe the initial data, instead of $c$. However, with the above physical meaning it seems to be more favorable to choose $c$ than other parameters. 


$$
M_{\mathrm{AH}}=\frac{(n-2) V_{n-2}}{2 \kappa} r_{\mathrm{AH}}^{n-3}=\frac{(n-2) V_{n-2}}{2 \kappa} x^{n-3} y_{\mathrm{AH}}^{n-3}=\frac{(n-2) c^{1 / 2}}{2 \kappa} V_{n-2} x^{n-3} .
$$

Clearly, on the apparent horizon the mass becomes unbounded as $x \rightarrow \infty$. The spacetime fails to be asymptotically flat for supercritical case. As a result, the total mass of the black hole cannot be written in power-law form in terms of initial data. This divergence is an unnatural artifact of self-similarity. In the self-similar solutions this kind of divergence is unavoidable. In order to avoid this divergence one has to consider the influx of the scalar field with a finite duration only. This is equivalent to introducing a cutoff $x_{c}$ for the $x$-coordinate. Since this is not allowed in the strict sense of a self-similar solution, we just suppose that any behavior of fields in the outer region beyond the boundary $x_{c}$ does not seriously disturb the self-similar evolution described by the action (3). One may then interpret the solution as a nearly self-similarly collapsing (at least in the inner region of $x<x_{c}$ ) solution with a finite mass of

$$
\frac{M_{\mathrm{BH}}}{M_{*}}=\frac{(n-2) c^{1 / 2}}{16 \pi} V_{n-2}\left(\frac{x_{c}}{\ell_{*}}\right)^{n-3} .
$$

On the other hand, in Ref. [18] a more realistic model that represents such type of collapse in four spacetime dimension is constructed by a cut and paste method: cutting the spacetime along a null hypersurface and then joining it to an exterior solution (e.g., an outgoing Vaidya solution). The mass of the black hole is finite and always takes the form $M_{\mathrm{BH}} \propto\left(c-c^{*}\right)^{1 / 2}$. We easily expect from the symmetry of the system that a simple extension of cut and paste method of Ref [18] to higher dimensions leads to a similar result

$$
M_{\mathrm{BH}} \propto\left(c-c^{*}\right)^{\gamma}
$$

where $\gamma \equiv(n-3) / \sqrt{2(n-2)}$ is the scaling exponent and it was identified in Ref. 21.

It is interesting to observe that the critical value $c_{n}^{*}$ depends only on the spacetime dimension $n$, and significantly and monotonically decreases as the number of dimensions increases as shown in Eq.(9) and Figure 1. The value $y_{\mathrm{AH}}$ also increases as the number of dimensions increases. These facts tell that the black hole window for initial data enlarges as the spacetime dimension increases, because the parameter $c$ specifies the initial distribution of the massless scalar field. Let us consider a system collapsing from a given initial distribution of the scalar field specified by a value $c$ smaller than the critical value $c_{n}^{*}$ in $n$-dimensional spacetime. A black hole can then be formed through the gravitational collapse from the initial distribution in higher than $n$ dimensions, while with the same initial distribution the scalar field will disperse leaving behind just a flat spacetime without forming a black hole in $n$ or lower dimensions. Thus, we can conclude that higher dimensions favor a black hole rather than a flat spacetime with dispersive scalar field when the massless scalar field self-similarly collapses.

Even though we have considered only the self-similarly collapsing scalar field to study the effect of increase in dimensionality on the results of gravitational collapse, we would expect similar effects for the dimensional dependence of black hole formation in different systems from the universality of the critical phenomena. In fact, it was already observed in a few different systems. The authors of Ref. [26] investigated self-similar gravitational collapse of null dust fluid in higher-dimensional spacetimes and showed that as the number 


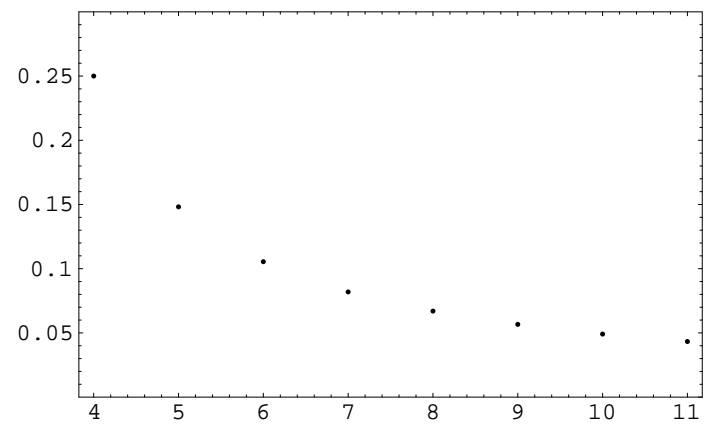

(a) $c^{*}$

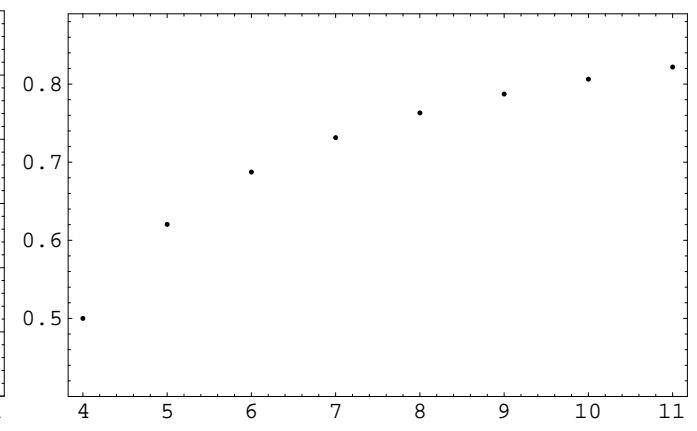

(b) $y_{\mathrm{AH}}$

FIG. 1. Values of $c^{*}$ and $y_{\mathrm{AH}}$ for critical collapse in various dimensions.

of dimensions increases window for naked singularity shrinks, that is, higher dimensions favor black hole rather than naked singularity. This could be seen from the fact that $\lambda_{c}$ decreases significantly as the dimension of the spacetime increases, where $\lambda_{c}$ is the critical value of the control parameter $\lambda$ which specifies the initial distribution of the null fluid as the parameter $c$ in our case. $\lambda_{c}$ depends on the spacetime dimension $n$ also and has the same value with $c^{*}$ as $\lambda_{c}=(n-3)^{n-3} /(n-2)^{n-2}$. P W While the final outcome of the collapse is a naked singularity if $\lambda \leq \lambda_{c}$, it is a black hole if $\lambda \geq \lambda_{c}$. This shows shrinking of the naked singularity window for initial data. A similar behavior has also been shown in the gravitational collapse of an inhomogeneous dust cloud described by higher-dimensional Tolman-Bondi spacetimes in Ref. [28], in that the effect of extra dimensions appears to be a shrinking of the naked singularity initial data space (of 4D) or an enlargement of the black hole initial data space, which is characterized by the parameter $\zeta_{0}$ there.

\section{QUANTUM COLLAPSE IN HIGHER DIMENSIONS}

Black holes are well understood as general-relativistic objects when their mass $M_{\mathrm{BH}}$ far exceeds the higher-dimensional gravity scale $M_{*}$. As $M_{\mathrm{BH}}$ approaches $M_{*}$, the black holes will become quantum gravitational objects and their properties become complex. This gives rise to an obstacle in calculating the production of black hole or analyzing the gravitational collapse. Hence, in the small mass limit it would be physically interesting and important to study the quantum gravity effect in the collapse. From the mass formulas Eqs.(11) and (12), we see that there are two independent small mass limits, $x_{c} \rightarrow \ell_{*}$ and $c \rightarrow c^{*}$. In the case $x_{c} \sim \ell_{*}$ the black hole mass is of the order $M_{*}$. As $c \rightarrow c^{*}$ it vanishes, so the classical description at the final stage would not be valid.

It is the purpose of this section to study quantum mechanically the black hole formation and to investigate how quantum gravity effects modify the classical picture and the dimensional dependence of gravitational collapse in higher dimensions. We use the ArnowittDeser-Misner (ADM) formulation to quantize the model and analyze the black hole wave function. In order to canonically quantize the system, we start from setting $\sigma=0$ in the

\footnotetext{
${ }^{3}$ Here we have redefined $\lambda$ according to our convention, so this is different by the factor $2^{-(n-2)}$ from that appears in Ref. 26].
} 
metric Eq.(3) and introduce a lapse function $N(\tau)$ to write the metric as

$$
d s^{2}=-x^{2} N^{2}(\tau) d \tau^{2}+d x^{2}+x^{2} y^{2}(\tau) d \Omega_{n-2}^{2}
$$

With this metric the action (3) is rewritten after taking into account the boundary term as

$$
S=V_{n-2} \ell_{*}^{n-2} \int d \chi d \tau N y^{n-4}\left[\frac{(n-3)}{2 \kappa}\left(-\frac{\dot{y}^{2}}{N^{2}}-y^{2}+1\right)+\frac{y^{2}}{2(n-2)} \frac{\dot{\phi}^{2}}{N^{2}}\right] .
$$

The canonical momentum densities of this system for $y$ and $\phi$ are defined as

$$
\begin{aligned}
& \pi_{y}=\frac{\partial \mathcal{L}}{\partial \dot{y}}=-\frac{n-3}{\kappa} \frac{y^{n-4} \dot{y}}{N} \\
& \pi_{\phi}=\frac{\partial \mathcal{L}}{\partial \dot{\phi}}=\frac{1}{(n-2)} \frac{y^{n-2} \dot{\phi}}{N} .
\end{aligned}
$$

Then the action $S$ can be rewritten in the ADM formulation as

$$
S=V_{n-2} \ell_{*}^{n-2} \int d \tau d \chi\left[\pi_{y} \dot{y}+\pi_{\phi} \dot{\phi}-N \mathcal{H}\right]
$$

where $\mathcal{H}$ is the effective superspace Hamiltonian density. The lapse function acts as a Lagrange multiplier, so varying the action with respect to $N$ we get the Hamiltonian constraint: $H=0$, where

$$
\begin{aligned}
H & \equiv V_{n-2} \ell_{*}^{n-2} \int d \chi \mathcal{H} \\
& =V_{n-2} \ell_{*}^{n-2} \int d \chi\left\{-\frac{\kappa}{2(n-3)} \frac{\pi_{y}^{2}}{y^{n-4}}+\frac{n-2}{2} \frac{\pi_{\phi}^{2}}{y^{n-2}}-\frac{(n-3)}{2 \kappa}\left(1-y^{2}\right) y^{n-4}\right\}
\end{aligned}
$$

Following Dirac's quantization method, the Hamiltonian constraint becomes a quantum constraint on the wave function

$$
\hat{H}\left(y, \pi_{y} ; \phi, \pi_{\phi}\right) \mid \Psi>=0 .
$$

The Hamiltonian constraint is then reduced to

$$
\left[-\frac{\kappa}{2(n-3) K} \frac{\hat{\Pi}_{y}^{2}}{\hat{y}^{n-4}}+\frac{n-2}{2 K} \frac{\hat{\Pi}_{\phi}^{2}}{\hat{y}^{n-2}}-\frac{(n-3) K}{2 \kappa}\left(1-\hat{y}^{2}\right) \hat{y}^{n-4}\right] \mid \Psi>=0,
$$

where we have defined the canonical momenta $\hat{\Pi}_{i} \equiv V_{n-2} \ell_{*}^{n-2} \int d \chi \hat{\pi}_{i}=K \hat{\pi}_{i}$, and $K \equiv$ $V_{n-2} \ell_{*}^{n-2} \int d \chi=V_{n-2} x^{n-2} . K$ represents the volume of $(n-2)$-dimensional sphere with radius $x$. It has an infinite volume in the limit $x \rightarrow \infty$, and the canonical momenta and the Hamiltonian are divergent. This divergence is an unnatural artifact of self-similarity as the black hole mass also diverges. In order to avoid this divergent Hamiltonian we have to consider the influx of scalar field with finite duration only, as done to avoid the divergent black hole mass in the previous section, and we introduce a cutoff $x_{c}$ in the $x$-coordinate. This cutoff is related to a scale of the inner region where the assumption of self-similarity 
is valid. Since the large $x_{c}$ limit is the large black hole limit, it will be equivalent to the classical limit.

When we quantize the system, the first term of equation (19) contains an ordering ambiguity between operators $\hat{y}$ and $\hat{\Pi}_{y}$. For many choices of the factor ordering, the effect of the factor ordering can be parameterized by a constant $a$, and the corresponding Hamiltonian is obtained by the substitution of $\Pi_{y}^{2} \rightarrow-y^{-a}\left[\partial_{y} y^{a} \partial_{y}\right]$. However, in this paper we do not adhere to the generality of the operator ordering, and for simplicity we take the simplest ordering, i.e., $a=0$. Of cause, due to this-like free fixing of the operator ordering the validity of our result may be limited. However, we expect that the factor ordering does not affect any of the semiclassical calculations done in this paper.f We will let the choice of $a$ be dictated by convenience. The Wheeler-De Witt (WDW) equation (19) then becomes

$$
\left[-\left(\frac{\partial}{\partial y}\right)^{2}+\frac{(n-2)(n-3)}{\kappa y^{2}}\left(\frac{\partial}{\partial \phi}\right)^{2}+\frac{(n-3)^{2} K_{c}^{2}}{\kappa^{2}}\left(1-y^{2}\right) y^{2(n-4)}\right] \Psi(y, \phi)=0 .
$$

where $K_{c} \equiv V_{n-2} x_{c}^{n-2}$. The wave function can be separated into the scalar field and gravitational field parts as

$$
\Psi(\phi, y)=e^{ \pm i \Pi_{\phi} \cdot \phi} \Phi(y) .
$$

Then the Wheeler-De Witt equation reduces to one-dimensional Schrödinger type equation

$$
\left[-\frac{1}{2}\left(\frac{\partial}{\partial y}\right)^{2}+V_{W D W}(y)\right] \Phi(y)=0,
$$

where the quantum mechanical potential $V_{W D W}(y)$ is given by

$$
V_{W D W}(y)=\frac{(n-3)^{2} K_{c}^{2}}{2 \kappa^{2}} y^{2(n-4)}\left[1-y^{2}-c\left(\frac{1}{y}\right)^{2(n-3)}\right],
$$

with $c$ related to the conjugate momentum density $\pi_{\phi}$ by $c \equiv[(n-2) /(n-3)] \kappa \pi_{\phi}^{2}$. As easily observed from Eqs. (田) and (15) $c$ is the same parameter with that used in the previous section. This potential is different from its classical counter part (23) by the multiplication factor $\left[(n-3)^{2} K_{c}^{2} / \kappa^{2}\right] y^{2(n-4)}$. The shape of the potential in each dimension depends on two parameters, the initial data $c$ and the cutoff $x_{c}$. As in the classical collapse, $c$ determines the supercritical $\left(c>c^{*}\right)$, the critical $\left(c=c^{*}\right)$ and the subcritical $\left(0<c<c^{*}\right)$ collapses. The cutoff $x_{c}$ makes the top of the potential shift away from zero.

In the classical collapse, the collapsing system was replaced by the system of a particle living in $y$ space with zero energy under the influence of the potential $V_{c l}$, and the collapse

4 For arbitrary $a$, taking $\partial_{Y} \equiv y^{a} \partial_{y}$ the WDW equation (22) is written as $\left[-(1 / 2) \partial_{Y}^{2}+\right.$ $\left.\bar{V}_{W D W}\right] \bar{\Phi}(Y)=0$, where $\bar{V}_{W D W}(Y) \equiv y^{2 a} V_{W D W}(y)$. On the other hand, as we will see soon after in the subsection 3. A, the black hole formation rate depends on the factor $\alpha \equiv \int d y \sqrt{\left|V_{W D W}(y)\right|}$. If we would calculate it with the semiclassical solution $\bar{\Phi}(Y)$ instead of $\Phi(y)$, then the rate would depend on $\bar{\alpha} \equiv \int d Y \sqrt{\left|\bar{V}_{W D W}(Y)\right|}$. However, we can easily see that $\alpha=\bar{\alpha}$. Therefore, the factor ordering does not affect any of the semiclassical calculations. 
parallels to the motion of a particle which starts from the infinity $(y=\infty)$ and rolls toward zero $(y=0)$. On the other hand, the Wheeler-De Witt equation (22) looks like a Schrödinger equation, and wave functions which satisfy the WDW equations describe a particle living in $y$ space under the influence of the potential $V_{W D W}$. Therefore, from experiences in quantum mechanics it seems to be plausible to replace the quantum collapse with the scattering problem of a wave function living in $y$ space. The boundary condition for the wave functions for black hole formation then is such that the wave function should be incident from the spatial infinity in $y$ space, i.e., $y=\infty$, and some part of them be reflected by the potential barrier back to the infinity of $y$ but the remaining part be transmitted toward the black hole singularity inside apparent horizon. With armed these boundary conditions, we compute the rate for black hole formation.

Unfortunately, it is impossible to find exact solutions of the Wheeler-De Witt equation (22) except for $n=4$. Therefore, we will try to see some aspects of quantum collapse by using the WKB method in several limiting cases: i) $x_{c} \gg \ell_{*}$ and $c \ll c^{*}$, ii) $c \sim c^{*}$. Here, $x_{c} \gg \ell_{*}$ means that $x_{c}$ is large enough for the top of the potential to be far from zero, so that the WKB is valid near the top of the potential.

\section{A. Subcritical collapse: $x_{c} \gg \ell_{*}$ and $c \ll c^{*}$}

In the case of subcritical collapse, the potential $V_{W D W}$ in any dimension has two zeros which we will call $y_{n 1}$ and $y_{n 2}\left(>y_{n 1}\right)$. We then define three separated regions, I $\left(0<y<y_{n 1}\right)$, II $\left(y_{n 1}<y<y_{n 2}\right)$ and III $\left(y>y_{n 2}\right)$. In this regime we assume $c$ is small enough compared to $c^{*}$ and $x_{c}$ large enough, so that the semi-classical analysis valid. Since we are interested in the collapse of the scalar field starting from $y=\infty$, we assume that the wave function has a purely incoming flux toward the singularity at $y=0$ in region $\mathrm{I}$, and the current of incident wave function in region III is unity for convenience. With these boundary conditions, the WKB wave functions in each region are given by

$$
\begin{aligned}
\Phi_{\mathrm{I}}(y) & =\frac{i}{\sqrt{p} \cosh \alpha} \exp \left[i \int_{y}^{y_{1}} p d y-\frac{\pi}{4} i\right], \\
\Phi_{\mathrm{II}}(y) & =\frac{i}{2 \sqrt{p} \cosh \alpha}\left[\exp \left(-\int_{y_{1}}^{y} p d y\right)-i \exp \left(\int_{y_{1}}^{y} p d y\right)\right], \\
\Phi_{\mathrm{III}}(y) & =\frac{1}{\sqrt{p}} \exp \left(-i \int_{y_{2}}^{y} p d y-\frac{\pi}{4} i\right)+\frac{\tanh \alpha}{\sqrt{p}} \exp \left(i \int_{y_{2}}^{y} p d y+\frac{\pi}{4} i\right),
\end{aligned}
$$

where $p \equiv \sqrt{2\left|V_{W D W}\right|}$ and

$$
\alpha \equiv \int_{y_{1}}^{y_{2}} p d y=\frac{(n-3) K_{c}}{\kappa} \int_{y_{1}}^{y_{2}} d y y^{(n-4)}\left|1-y^{2}-c y^{-2(n-3)}\right|^{1 / 2} .
$$

Except for the phase factor, the wave function (24) depends only on $\alpha$ which determines the tunnelling and reflection amplitudes of the waves. The wave function $\Phi_{\text {III }}$ indeed satisfies the boundary condition for black hole formation, in that the first term describes the incoming component and the second term describes the outgoing (reflected) component at the far region $(y \gg 1)$. 
The existence of nonvanishing wave $\Phi_{\mathrm{I}}$ in region I implies that there is a possibility to form a black hole quantum mechanically, even if it is classically forbidden. We now calculate the rate of a black hole formation in the subcritical collapse. The incoming flux at spatial infinity computed with Eq.(26) is

$$
j_{\text {in }}(y)=\operatorname{Im}\left[\Phi_{\text {in }}^{*}(y) i \hat{\Pi}_{y} \Phi_{\text {in }}(y)\right] \rightarrow 1 .
$$

Some part of the incoming flux is reflected by the potential barrier back to the infinity $y=\infty$ but the remaining part transmits toward the black hole singularity at $y=0$. On the other hand, the reflection rate at the infinity is similarly found as

$$
P_{\mathrm{R}}=\frac{j_{\mathrm{re}}}{j_{\text {in }}}=\tanh ^{2} \alpha
$$

The rate for black hole formation is the ratio of the transmitted flux to the incident flux. From the flux conservation we obtain the rate for black hole formation

$$
P_{\mathrm{T}}=\frac{j_{\mathrm{tr}}}{j_{\mathrm{in}}}=1-\frac{j_{\mathrm{re}}}{j_{\mathrm{in}}}=\operatorname{sech}^{2} \alpha
$$

This transmission rate coincides with the rate near $y=0$ obtained by a direct computation using the transmitted wave Eq.(24).

As expected previously, the classical limit is obtained in the large limit of $K_{c}$ (or equivalently $\left.x_{c}\right)$. In a spacetime of dimension $n, \alpha$ depends only on the cut off as $\propto\left(x_{c} / \ell_{*}\right)^{n-2}$. As $\alpha$ increases the transmission rate $P_{\mathrm{T}}$ exponentially decreases and vanishes, while the reflection rate $P_{\mathrm{R}}$ approaches to unity. Therefore, most part of the scalar field bounces back without forming a black hole in the limit of large $x_{c}$.

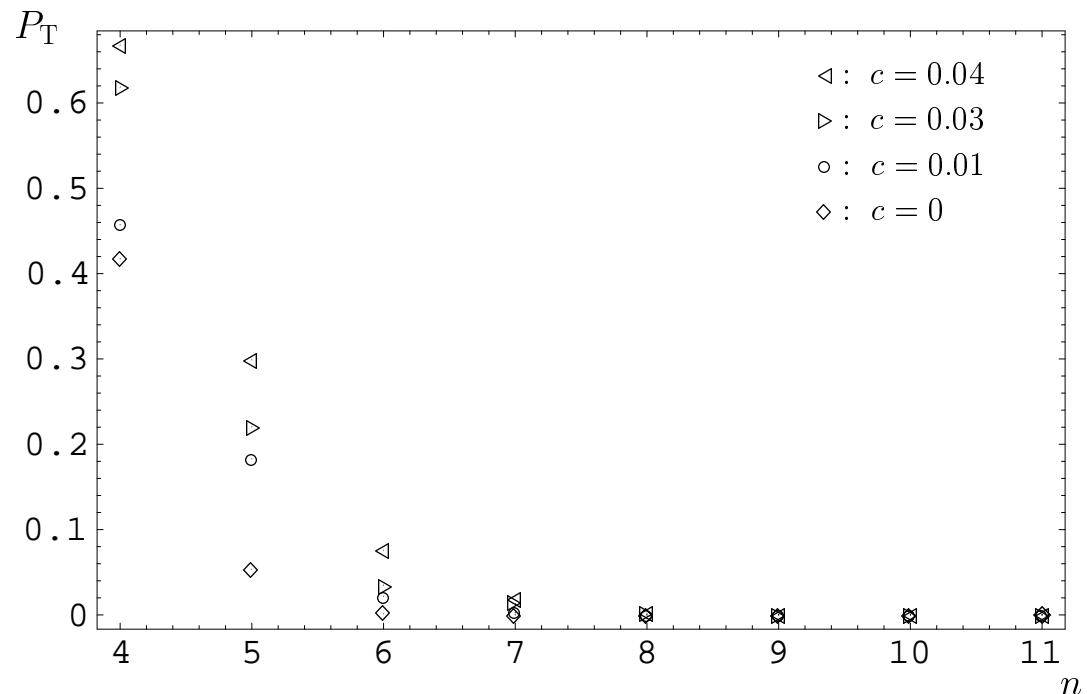

FIG. 2. The rate for black hole formation in diverse dimensions for various values of the control parameter $c$ and a value of the cutoff $x_{c}=1.6 \ell_{P l}$.

On the other hand, the value of $\alpha$ increases as $c$ decreases, and has a maximum value when $c=0$ for a given cutoff $x_{c}$ and dimension $n$. The rate for black hole formation falls off as $c$ decreases, and closes to the minimum rate in the limit $c \rightarrow 0$ : 


$$
P_{\mathrm{T}, \min } \rightarrow \operatorname{sech}^{2}\left[\frac{K_{c}}{\kappa} \frac{\sqrt{\pi} \Gamma\left(\frac{n-1}{2}\right)}{2 \Gamma\left(\frac{n}{2}\right)}\right] \quad \text { as } \quad c \rightarrow 0
$$

Since the parameter $c$ characterizes the gravitational interaction strength in the collapse and small $c$ means small interaction strength, it is reasonable that the rate for black hole formation falls off as $c$ becomes small. However, it seems surprising that there exists a minimum rate for black hole formation with vanishingly small gravitational interaction strength of limit $c \rightarrow 0$. This is not an artifact of our approximation by WKB method. We can also observe this aspect in Ref. [30], in which the transmission rate is exactly calculated using the analytic solution of Eq. (22). Unless $c=0$ definitely, the potential is singular at the origin at $y=0$, and the incident waves are not reflected at the origin. 1 Note that the curvature singularity at the origin acts like a sink for the incident waves. Therefore, the transmission rate does not become zero in the limit $c \rightarrow 0$. The minimum rate may have a different form from Eq. (31), because in the limit $c \rightarrow 0$ the potential becomes very flat near $y=y_{n 1}$ and is very close to zero in a broad range. So our WKB approximation would not be valid near $y=y_{n 1}$. The numerical works in Fig. 1 confirm the nonvanishing transmission rate in the limit $c \rightarrow 0$. On the other hand, we have to consider the position of the apparent horizon. If the apparent horizon is at $y=0$ as in the classical collapse, then there is no black hole formation despite of the existence of the transmission flux in the region I. However, the apparent horizon and black hole mass will be affected by quantum effect. The quantum mechanical effect will shift the position of the apparent horizon from $y=0$ to $y \sim 1 / K_{c}^{2}$ as discussed in Ref. 30].

The rate for black hole formation doesn't seem to vary simply with spacetime dimensions for a fixed initial data $c$ and cutoff $x_{c}$. The $n$ dependence of $\alpha$ is complicated as can be seen from Eq.(27). The volume of $(n-2)$-dimensional unit sphere $V_{n-2}$ included in $K_{c}$ does not monotonically behave but has a maximum value at $n=7$, while the other factors monotonically increase as $n$ increases. However, with some generic values of the cutoff $x_{c}, \alpha$ behaves simply and $P_{\mathrm{T}}$ monotonically decreases within four to eleven dimensions, in which we are interested from the unified theory perspective like string theory. Since the integral in Eq.(27) is not explicitly calculable in general dimensions, we present numerical values of $P_{\mathrm{T}}$ for several different $c$ in Figure 2. The result shows that the rate for black hole formation by quantum tunneling falls off as the number of dimensions increases for a given initial data $c$ and fixed cutoff $x_{c}$.

\section{B. Near-critical collapse: $c \sim c^{*}$}

In this regime we cannot directly apply the usual WKB approximation, because the height of the potential is too low and the derivative of the potential is almost zero near turning points. So the semiclassical solution such as $\Phi_{\text {II }}$ of previous case is not valid near the top of

\footnotetext{
${ }^{5}$ The case $c=0$ should be distinguished from the limit $c \rightarrow 0$. If $c=0$ definitely, the potential is not singular but is zero at $y=0$. The incident waves are totally reflected at the origin, so the transmission rate is then zero. Notice that the spacetime described by the metric Eq. (2) does not have a singularity, but is regular at the origin.
} 
the potential. Note that WKB is valid only when the condition $\delta \lambda / \lambda \ll 1$ is satisfied, where $\lambda$ is the wave length of the wave functions. The spirit of the WKB method is that one find a solution of the wave equation near the turning point by approximating the potential with a linear function. Then one matches the solution with the other two semiclassical solutions outside the transition region.

We develop an approximation which has a common spirit with the usual WKB method. We approximate the potential $V_{W D W}$ with a quadratic function near the position of the top and find an analytic solution of the wave equation with the quadratic potential. We then match the solution with the other two semiclassical solutions in regions I and III choosing appropriate coefficients. This approximation method is not so different from the conventional WKB method but only a slight deformation from the WKB method, besides that we introduce a quadratic approximation instead of a linear approximation. So it has much common sprit with the usual WKB method, and it will give approximately good answer as much as the usual WKB method does.

We represent the near critical limit by setting $c=c^{*}(1+\delta)$ with $|\delta| \ll 1$, where the collapse is supercritical if $\delta>0$ and subcritical if $\delta<0$. The Wheeler-De Witt potential is then separated as

$$
V_{N C}(y)=V_{\text {crit }}(y)-\frac{(n-3)^{2} K_{c}^{2}}{2 \kappa^{2} y^{2}} c^{*} \delta
$$

where $V_{\text {crit }}(y)$ is potential for critical collapse and can be written as

$$
V_{\text {crit }}(y)=-\frac{(n-3)^{2} K_{c}^{2}}{2 \kappa^{2}}\left(y^{2}-y^{* 2}\right)^{2} \sum_{k=0}^{n-4} \frac{k+1}{n-2} y^{* 2(n-k-5)} y^{2 k-2} .
$$

$V_{\text {crit }}(y)$ has a second order zero at $y^{*}=\sqrt{(n-3) /(n-2)}$ as the classical potential $V_{\text {cl }}$ does. Near $y=y^{*}$, the potential may be approximated as

$$
\begin{aligned}
V_{N C} & \simeq-\frac{(n-3)^{2} K_{c}^{2}}{2 \kappa^{2} y^{2}}\left[c^{*} \delta+\frac{n-3}{2} y^{* 2(n-5)}\left(y^{2}-y^{* 2}\right)^{2}\right] \\
& =E-\frac{V_{+}}{2} y^{2}-\frac{V_{-}}{2 y^{2}}
\end{aligned}
$$

where

$$
\begin{aligned}
V_{-} & =\frac{(n-3)^{3} K_{c}^{2}}{2 \kappa^{2}} y^{* 2(n-3)}\left[1+\frac{2 c^{*} \delta}{n-3} y^{*-2(n-3)}\right], \\
V_{+} & =\frac{(n-3)^{3} K_{c}^{2}}{2 \kappa^{2}} y^{* 2(n-5)} \\
E & =\frac{(n-3)^{3} K_{c}^{2}}{2 \kappa^{2}} y^{* 2(n-4)}
\end{aligned}
$$

In fact, there could be several other choices in choosing the approximate quadratic potential. But we have chosen above approximate potential upon the following advantages: First, with this choice the WDW equation is exactly solvable. Second, it well approximates the full potential near the origin at $y=0$ as well as around the top, so the solutions for the 
approximate potential behaves like $\Phi_{I}$ in the region I. Lastly, in 4-dimensional case, it corresponds to the exact potential, not approximate one.

With above approximate potential, the Wheeler-De Witt equation allows solutions in terms of the confluent hypergeometric function [35:

$$
\Phi_{\mathrm{NC}}(y)=D e^{-i \sqrt{V_{+}} y^{2} / 2}\left(i \sqrt{V_{+}} y^{2}\right)^{\mu} M\left(a, b, i \sqrt{V_{+}} y^{2}\right)
$$

where $D$ is a constant and

$$
\begin{aligned}
\mu & \equiv \frac{1}{4}-\frac{i}{2} Q, \\
a & \equiv \frac{1}{2}-\frac{i}{2}\left(Q+\sqrt{E} y^{*}\right), \\
b & \equiv 1-i Q,
\end{aligned}
$$

with $Q=\left(E y^{* 2} \nu^{2}-1 / 4\right)^{1 / 2}, \nu=V_{-} /\left(E y^{* 2}\right)$. There exists another independent solution of the Wheeler-De Witt equation with the potential (34). However, we will not write down that solution, because the solution (38) is enough to describe the formation of a black hole. Remind that we follow the analogy to the scattering problem of quantum mechanics and the boundary conditions mentioned previously. According to the boundary conditions, it seems reasonable to choose only purely ingoing wave functions in region I. The same wave function was used to describe the four-dimensional formation of a black hole by a scalar field collapse 30.

We now match this solution with the semiclassical solutions in regions I and III. In fact, the solution (38) is also valid near $y=0$, because the approximate potential $V_{N C}$ has the same asymptotic form as the full potential $V_{W D W}$. Therefore, we only need to match the solution (41) with the semiclassical potential in region III. Using the asymptotic form of the confluent hypergeometric function

$$
\lim _{|z| \rightarrow \infty} M(a, b, z) \approx \Gamma(b)\left[\frac{e^{i \pi a} z^{-a}}{\Gamma(b-a)}+\frac{e^{z} z^{a-b}}{\Gamma(a)}\right], \quad-\frac{\pi}{2}<\arg (z)<\frac{3 \pi}{2},
$$

we get the asymptotic form of the wave function at large $y$ :

$\Phi_{\mathrm{NC}}(y) \approx D\left\{\frac{\Gamma(b)}{\Gamma(b-a)} e^{i \pi a}\left(i \sqrt{V_{+}} y^{2}\right)^{\mu-a} e^{-i \sqrt{V_{+}} y^{2} / 2}+\frac{\Gamma(b)}{\Gamma(a)}\left(i \sqrt{V_{+}} y^{2}\right)^{\mu+a-b} e^{i \sqrt{V_{+}} y^{2} / 2}\right\}$,

where $a-\mu=1 / 4-i \sqrt{E} y^{*} / 2$ and $b-a-\mu=1 / 4+i \sqrt{E} y^{*} / 2$. The first term describes the incoming wave function and the second term describes the outgoing wave function. Comparing the asymptotic form (41) with the semiclassical potential in region III of the form

$$
\Psi(y)=\frac{D_{1}}{\sqrt{p(y)}} e^{i \int^{y} p\left(y^{\prime}\right) d y^{\prime}}+\frac{D_{2}}{\sqrt{p(y)}} e^{-i \int^{y} p\left(y^{\prime}\right) d y^{\prime}},
$$

we obtain the asymptotic form of the wave function in the region III

$$
\Psi_{\mathrm{III}}(y) \approx \tilde{D}\left\{\frac{\Gamma(b) e^{i \pi a}}{\Gamma(b-a)}\left[\frac{\sqrt{V_{+}}}{p^{2}(y)}\right]^{a-\mu} e^{-i \int^{y} p\left(y^{\prime}\right) d y^{\prime}}+\frac{\Gamma(b)}{\Gamma(a)}\left[\frac{\sqrt{V_{+}}}{p^{2}(y)}\right]^{b-a-\mu} e^{i \int^{y} p\left(y^{\prime}\right) d y^{\prime}}\right\}
$$


where $p=\sqrt{2 V_{W D W}}$. Note that the asymptotic solution (41) is of the same form as the semiclassical solution (42) with $\bar{p} \equiv \sqrt{-2 V_{N C}}$.

Then the rate for black hole formation is easily obtained from the two solutions (41) and (43):

$$
P_{\mathrm{T}}=\frac{j_{\mathrm{tr}}}{j_{\mathrm{in}}}=\frac{e^{-\frac{\pi}{2}\left(\sqrt{E} y^{*}+Q\right)} \sinh \pi Q}{\cosh \frac{\pi}{2}\left(\sqrt{E} y^{*}-Q\right)} .
$$

It is easy to check that this transmission rate coincides with that obtained by using current conservation at the spatial infinity

$$
P_{\mathrm{R}}=\frac{j_{\mathrm{re}}}{j_{\text {in }}}=\frac{\cosh \frac{\pi}{2}\left(Q+\sqrt{E} y^{*}\right)}{\cosh \frac{\pi}{2}\left(Q-\sqrt{E} y^{*}\right)} e^{-\pi Q}=1-\frac{j_{\mathrm{tr}}}{j_{\text {in }}} .
$$

This determines the probability for black hole formation in the near critical collapse for values of $|\delta| \ll 1$.

In the large cutoff limit $x_{c} \gg \ell_{*}$ it will be easier to see the behavior of the rate $P_{\mathrm{T}}$. In the large $x_{c}$ limit the rate for black hole formation should be calculated separately for $\delta>0$ and $\delta<0$. For $\delta>0$, Eq.(44) becomes asymptotically

$$
P_{\mathrm{T}} \approx 1-\exp \left[-\frac{\sqrt{2} \delta K_{c}}{\sqrt{n-3} \kappa}\left(\frac{n-3}{n-2}\right)^{\frac{n}{2}}\right] .
$$

In the case $\delta<0$, the asymptotic value of Eq.(44) in the large $x_{c}$ limit is

$$
P_{\mathrm{T}} \approx \exp \left[\frac{\sqrt{2} \delta K_{c}}{\sqrt{n-3} \kappa}\left(\frac{n-3}{n-2}\right)^{n / 2}\right] .
$$

This is the tunneling rate for black hole formation in the subcritical collapse near the critical case. The case that $\delta=0$ has to be considered separately. The asymptotic form is then

$$
P_{\mathrm{T}}=\frac{e^{-\frac{\pi}{2}\left(\sqrt{V_{-}}+\sqrt{V_{-}-1 / 4}\right)} \sinh \pi \sqrt{V_{-}-1 / 4}}{\cosh \frac{\pi}{2}\left(\sqrt{V_{-}}+\sqrt{V_{-}-1 / 4}\right)} .
$$

As in the previous case, the classical limit is obtained in the very large cutoff limit $x_{c} \gg \ell_{*}$. The rate $P_{\mathrm{T}}$ exponentially depends on $K_{c}$. In the very large $x_{c}$ limit $P_{\mathrm{T}}$ becomes unity for $\delta>0$, which implies that a black hole is formed from the collapsing scalar field as in the classical case. For $\delta<0, P_{\mathrm{T}}$ becomes zero. This tells that the scalar field bounces back without forming a black hole. For $\delta=0, P_{\mathrm{T}}$ becomes $1 / 2$.

On the other hand, the dimensional dependence of the rate $P_{\mathrm{T}}$ is more complicated, because the volume factor $V_{n-2}$ included in $K_{c}$ does not behave simply but reaches to the maximum value at $n=7$, while the product of all the other factors in the exponent of Eq.(46) monotonically increases with fixed $c$ and $x_{c}$ as dimension $n$ increases. However, for generic values of $x_{c}$ the absolute value of the exponent monotonically increases between four to eleven dimensions. As the spacetime dimension $n$ increases the rate for black hole formation $P_{\mathrm{T}}$ increases for $\delta>0$, while $P_{\mathrm{T}}$ decreases for $\delta<0$. Therefore, the rate for black hole 


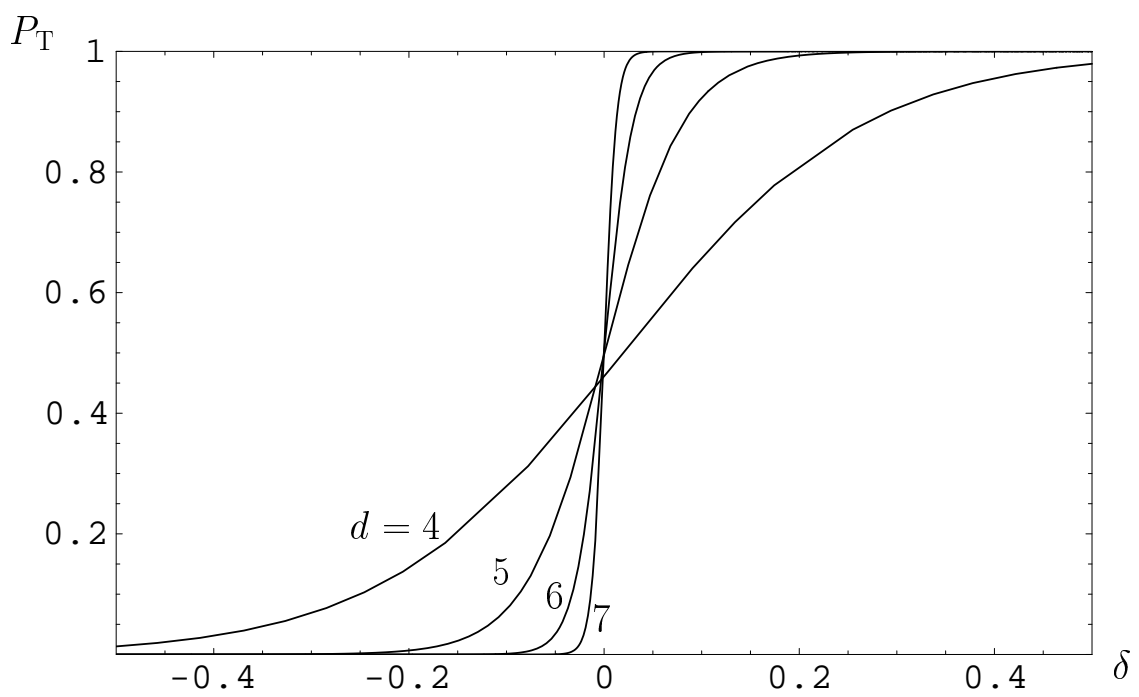

FIG. 3. The rates for black hole formations in near critical collapses in diverse dimensions for a value of the cutoff $x_{c}=1.5 \ell_{P l}$.

formation by quantum tunnelling falls off as dimension increases in the subcritical collapse with $\delta<0$, while black hole formation is easier in higher dimensions for $\delta>0$, as shown in Fig. 5. This implies that the aspect of quantum collapse becomes close to the classical aspect as dimension increases. This can be interpreted as the quantum gravity effects on the result of gravitational collapse lessens with increase of the spacetime dimensionality.

\section{DISCUSSIONS: GRAVITATIONAL COLLAPSE IN A BRANE WORLD WITH LARGE BUT COMPACT EXTRA DIMENSIONS}

We have considered self-similarly collapsing system of a massless scalar field in higher dimensions, both classically and quantum mechanically. We examined the dimensional dependence of such gravitational collapse and formation of black hole. Higher dimensional spacetime seems to favor formation of black hole rather than other final outcomes, in that the initial data space for black hole formation enlarges as dimension increases, i.e., the critical value $c_{n}^{*}$ of the control parameter which describes initial distribution of the scalar field falls off as dimension increases.

We have studied black hole formation quantum mechanically by the collapsing scalar field. We canonically quantized the system and found the wave functions by using the WKB method. The results of quantum collapse obtained in four-dimensional spacetime [30] go over to higher-dimensional quantum collapse. The wave functions for black hole formation have not only incoming flux but also outgoing flux in both the subcritical and supercritical cases. The classical evolution of black hole formation is recovered in the limit of large cutoff $x_{c}$. On the other hand, the rate for black hole formation by quantum tunnelling in the subcritical collapse lessens as spacetime dimension increases, that is, it becomes harder to form a black hole in higher dimension in the subcritical collapse. The reflection rate in the supercritical (but close to the critical) collapse, however, decreases as dimension increases: The black hole formation is easier in higher dimensions in the supercritical collapse. This implies that the quantum gravity effect on the gravitational collapse lessens with the increase 
of spacetime dimension.

We now discuss the gravitational collapse in a brane world with large but compact extra dimensions first explored by Arkani-Hamed et. al. [32]. The dimensional dependence of the final result in gravitational collapse implies that if we are living in the brane world with large extra dimensions, then the aspect of gravitational collapse is drastically different from that expected in usual four-dimensional world. Even though we prepare a macroscopic initial distribution of matter in four-dimensional spacetime, which final product is resulted from the collapse will depend on the number of extra dimensions, because if the Schwarzschild radius is smaller than the size of extra dimensions, the collapse will be higher-dimensional rather than four-dimensional at the final stage of the collapse.

Suppose that one prepares an initial distribution of the scalar field, of which typical size is much larger than the extra dimension size, so that the collapse is effectively fourdimensional at the early stage of the collapse. Here, we assume that the three-brane has negligible self-gravity of its own as in usual large extra dimension scenarios and so the brane can be identified as a surface of vanishing extrinsic curvature. Therefore, if we restrict to black holes of size $r_{\mathrm{BH}}$ much less than the size of extra dimensions $L$, then the geometry near the black hole will be very well approximated by a $n$-dimensional Schwarzschild solution [31. If a black hole is formed from matters on the brane, the symmetry requires that the brane pass through the equator of the black hole. If the initial distribution is prepared (taking $c>c_{4}^{*}$ and large enough $x_{c}$ ) to form a black hole with Schwarzschild radius $r_{\mathrm{BH}}$ larger than the extra dimension size $L\left(r_{\mathrm{BH}} \gg L\right)$, then the collapse will be effectively four-dimensional event to an observer outside the horizon, because the observer can not see the collapse after the apparent horizon forms. The geometry of the resulting black hole is simply a product of four-dimensional Schwarzschild solution and a $(n-4)$-dimensional torus, and its dynamical properties are determined by four-dimensional physics.

However, if the initial distribution is prepared (if a black hole is formed) so that the resulting black hole have Schwarzschild radius of $\ell_{*} \ll r_{\mathrm{BH}} \ll L$, the collapse goes under the size of the extra dimensions and becomes essentially $n$-dimensional at the final stage of the collapse. 5 Therefore, the final result of the collapse can be different from that expected in the purely four-dimensional world. If one prepares the initial distribution specified by $c>c_{4}^{*}$, then a black hole is formed as expected in four-dimensional world because $c_{4}^{*}>c_{n}^{*}$. Since, for $r \gg L$, the geometry of the resulting black hole is well approximated by the four-dimensional Schwarzschild black hole and the mass measured on the brane is the same as the mass in the bulk, it may look like a usual four-dimensional Schwarzschild black hole. However, it has different thermodynamic properties from those of a purely four-dimensional

\footnotetext{
${ }^{6}$ The mass of the black hole can still be much larger than the four-dimensional Planck scale and can be treated as a general relativistic object. In the usual large extra dimension scenario [32], the size of extra dimensions $L$ is related to the effective four-dimensional Planck scale $M_{\mathrm{Pl}}$ and the $n$-dimensional gravity scale $M_{*}$ by $L \sim M_{*}^{-1}\left(M_{\mathrm{Pl}} / M_{*}\right)^{2 /(n-4)}$, while the Schwarzschild radius of $n$-dimensional black hole is $r_{\mathrm{BH}}=M_{*}^{-1}\left[16 \pi M_{\mathrm{BH}} /(n-2) V_{n-2} M_{*}\right]^{1 /(n-3)}$. So the black hole mass of size $r_{\mathrm{BH}} \sim L$ is $M_{\mathrm{BH}} \sim M_{*}\left(M_{\mathrm{Pl}} / M_{*}\right)^{2(n-3) /(n-4)}$. If $M_{*}$ is of order a TeV, then $M_{\mathrm{BH}} \sim 10^{32(n-3) /(n-4)} M_{*} \sim 10^{32(n-3) /(n-4)-21} \mathrm{~g}$. This is much larger than the four-dimensional Planck mass, e.g., for $n=6, M_{\mathrm{BH}} \sim 10^{27} \mathrm{~g}$ and, for $n=10, M_{\mathrm{BH}} \sim 10^{16} \mathrm{~g}$, about the mass of the Earth and the typical mass of primordial black holes, respectively.
} 
black hole [33]. The Schwarzschild radius is larger than it would be for a four-dimensional black hole. This means that the temperature is lower, the evaporation rate is slower, and the horizon area (the entropy of the black hole) is larger. If $c<c_{n}^{*}$, then there is no black hole formation and the scalar field disperses back to the four-dimensional world. The spectrum of the dispersed scalar field will not be like that in a purely four-dimensional collapse, because the very light Kaluza-Klein modes with masses starting at $\sim 1 / L$ couples to the matter and some fraction of the energy radiate off of the brane into the bulk.

The case that the initial distribution is prepared to have the control parameter of $c_{n}^{*}<$ $c<c_{4}^{*}$ may be more interesting. Then, an $n$-dimensional black hole will be formed and there will be no dispersing scalar field. This is not the one expected if we are living in purely fourdimensional world. If our world is purely four-dimensional, or the extra dimension is very small and of the order of quantum gravity scale as taken in usual higher-dimensional unified theories, then there will be no black hole formation and the scalar field will disperse back to the four-dimensional world. Therefore, we can expect that one can probe the existence and determine the number of large extra dimensions by examining the gravitational collapse and the dimensional dependence of black hole formation, (at least) in principle.

Of course, we have over-simplified the problem. At the final stage when the collapse effectively occurs in $n$ dimensions, the collapse is not like the $n$-dimensional spherical collapse discussed in the previous sections. The collapse is not spherically symmetric and self-similar, even if we have prepared the initial distribution to be spherically symmetric and the collapse to occur self-similarly at the beginning, at which the collapse can be described by the four-dimensional general relativity. The collapse may look like that of a disk-like rather than a spherical distribution at the late stage, because the matter does live only on the three-brane. Therefore, the discussions in the previous two paragraphs suffer from lack of physical meaning. Despite of this poor physical meaning, the purpose we presented the discussions upon the over-simplified assumption is simply to illustrate the probing mechanism of the large extra dimensions. In this sense, the discussions in the two previous paragraphs seems to have its necessity. For rigorous treat, we have to consider the collapse of disk-like distribution of matters and the critical phenomena. However, it seems impossible to find analytic solutions for collapses of disk-like matter distributions. Some numerical simulations for such collapses may be helpful. But, this is beyond the scope of this paper.

In spite of all, we easily expect that the brane world scenario makes interesting predictions about the formation of small black holes. In particular, the existence of the large extra dimensions would affect on the formation of primordial black holes, which are formed as a result of the density fluctuation in the early universe. If there was large extra dimensions, then mass spectrum of the primordial black hole would not be like that predicted in purely four-dimensional theory. It will be interesting to investigate their detailed phenomenology.

\section{Acknowledgments}

This work was supported in part by BK21 Project of Ministry of Education (S.-H.M. and J.H.Y.) and by Korea Research Foundation under Project number 99-005-D00010 (H.-C.K. and J.H.Y.). 


\section{REFERENCES}

[1] M. W. Choptuik, Phys. Rev. Lett. 70, 9 (1993).

[2] A.M. Abrahams and C.R. Evans, Phys. Rev. Lett. 70, 2980 (1993); Phys. Rev. D49, 3998 (1994).

[3] C. R. Evans and J. S. Coleman, Phys. Rev. Lett. 72, 1782 (1994).

[4] T. Koike and T. Mishima, Phys. Rev. D 51, 4045 (1995); T. Koike, T. Hara, and S. Adachi, Phys. Rev. Lett. 74, 5170 (1995).

[5] C. Gundlach, Phys. Rev. D 54, 7353 (1996); ibid. 55, 6002 (1997).

[6] S. Hod and T. Piran, Phys. Rev. D 55, R440 (1997); ibid., 3485 (1997).

[7] C. Gundlach, Phys. Rev. D 55, 6002 (1997).

[8] R. S. Hamade, J. H. Horne, and J. M. Stewart, Class. Quant. Grav. 13, 2241 (1996).

[9] E.W. Hirschmann and D.M. Eardley, Phys. Rev. D 51, 4198 (1995); ibid. 52, 5850 (1995); D. Garfinkle, ibid., 51, 5558 (1995); R.S. Hamadé and J.M. Stewart,Class. Quant. Grav. 13, 497 (1996).

[10] T. Harada and H. Maeda, Phys. Rev. D 63, 084022 (2001).

[11] I. Olabarrieta and M.W. Choptuik, Critical phenomena at the threshold of black hole formation for collisionless matter in shperical symmetry, gr-qc/0107076.

[12] M. D. Roberts, Gen. Rel. Grav. 21, 907 (1989).

[13] P.R. Brady, Class. Quant. Grav. 11, 1255 (1994); Phys. Rev. D 51, 4168 (1995).

[14] Y. Oshiro, K. Nakamura, and A. Tomimatsu, Prog. Theor. Phys. 91, 1265 (1994).

[15] V. Husain, E.A. Martinez, and D. Nunez, Phys. Rev. D 50, 3783 (1994); J. Trachen, ibid. 50, 7144 (1994); T. Koike and T. Mishima, ibid. 51, 4045 (1995); T. Koike, T. Hara, and S. Adachi, Phys. Rev. Lett. 74, 5170 (1995).

[16] D. Maison, Phys. Lett. B 366, 82 (1996).

[17] H.P. de Oliveira and E.S. Cheb-Terrab, Class. Quant. Grav. 13, 425 (1996); T. Chiba and J. Soda, Prog. Ther. Phys. 96, 567 (1996).

[18] A. Wang and H.P. de Oliveira, Phys. Rev. D 56, 753 (1997).

[19] C. Gundlach, Phys. Rev. Lett. 75, 3214 (1995); Phys. Rev. D 55, 695 (1997).

[20] C. Gundlach, Adv. Theor. Math. Phys. 2, 1-49 (1998); Living Reviews 1999-4 (1999).

[21] J. Soda and K. Hirata, Phys. Lett. B 387, 271 (1996).

[22] A. V. Frolov, Class. Quant. Grav. 16, 407 (1999).

[23] L.K. Patel and N. Dadhich, Exact solutions for null fluid collapse in higher dimensions, gr-qc/9909068.

[24] J.F. Villas da Rocha and A.Z. Wang, Gravitational Collapse of Perfect Fluid in Ndimensional Spherically Symmetric Spacetime, gr-qc/9910109.

[25] D. Garfinkle, C. Cutler, and G. Comer Duncan, Phys. Rev. D 60, 104007 (1999).

[26] S.G. Ghosh and N. Dadhich, On naked singularity in higher dimensional Vaidya spacetime, gr-qc/0105085.

[27] J.F. Villas da Rocha, Type II fluid solutions to Einstein's field equations in $N$ dimensional spherical spacetime, gr-qc/0105095.

[28] S.G. Ghosh and A. Beesham, Higher dimensional inhomogeneous dust collapse and cosmic censorship, gr-qc/0108011.

[29] A. Tomimatsu, Phys. Rev. D 52, 4540 (1995).

[30] D. Bak, S.P. Kim, S.K. Kim, K.-S. Soh, and J.H. Yee, Phys. Rev. D 60, 064005 (1999), ibid. 61, 044005 (2000); ibid. 62, 047054 (2000). 
[31] R. Myers and M. Perry, Ann. Phys. (N.Y.) 172, 304 (1986).

[32] N. Arkani-Hamed, S. Dimopoulos, and G. Dvali, Phys. Lett. B 429, 263 (1998); Phys. Rev. D 59, 086004 (1999); I. Antoniadis, N. Arkani-Hamed, S. Dimopoulos, and G. Dvali, Phys. Lett. B 436, 257 (1998).

[33] R. Emparan, G.T. Horowitz, and R.C. Myers, Phys. Rev. Lett. 85, 499 (2000).

[34] S.B. Giddings and Thomas, High energy colliders as black hole factories: The end of short distance physics, hep-ph/0106219; S. Dimopoulos and G. Landsberg, Black Holes at the $L H C$, hep-ph/0106295.

[35] M. Abramowitz and I.A. Stegun, Handbook of Mathematical Functions (Dover, New York, 1972).

[36] J.P.S. Lemos, Phys. Rev. Lett. 68, 1447 (1992); Phys. Rev. D 59, 044020 (1999). 